\title{
Letters to the Editor : Stratégies d'utilisation par une communauté de chercheurs francophones et tentative de caractérisation du genre
}

Didier Carnet et Anne Magnet

\section{(2) OpenEdition}

Journals

Édition électronique

URL : http://journals.openedition.org/asp/1613

DOI : 10.4000/asp.1613

ISBN : 978-2-8218-0388-6

ISSN : 2108-6354

Éditeur

Groupe d'étude et de recherche en anglais de spécialité

\section{Édition imprimée}

Date de publication : 1 décembre 2002

Pagination : 89-102

ISSN : 1246-8185

Référence électronique

Didier Carnet et Anne Magnet, «Letters to the Editor: Stratégies d'utilisation par une communauté de chercheurs francophones et tentative de caractérisation du genre », ASp [En ligne], 35-36 | 2002, mis en ligne le 24 août 2010, consulté le 10 décembre 2020. URL : http://journals.openedition.org/asp/ 1613 ; DOI : https://doi.org/10.4000/asp.1613

Ce document a été généré automatiquement le 10 décembre 2020.

Tous droits réservés 


\title{
Letters to the Editor: Stratégies d'utilisation par une communauté de chercheurs francophones et tentative de caractérisation du genre
}

\author{
Didier Carnet et Anne Magnet
}

1 Cet article s'inscrit dans le cadre d'une étude plus générale entreprise sur un genre peu étudié à notre connaissance : les Letters to the Editor (Magnet \& Carnet 2001 : 51-62). En effet, le discours scientifique a surtout été analysé dans sa forme la plus courante et la plus répandue : l'article de recherche primaire. Cette forme de discours est un discours contraint, modélisé, et donc assimilé, à la suite des travaux de J. Swales (1990), à un genre dont les caractéristiques discursives commencent à être connues et les spécificités linguistiques reconnues sinon totalement décrites et analysées.

2 D'un point de vue épistémologique, l'article de recherche primaire représente la vision officielle, l'orthodoxie d'une communauté de spécialistes rendue implicite par le processus de peer-review. Le but de ce type d'article est dans une majorité de cas de venir conforter, confirmer le paradigme du moment (Martin 1996: 15). Cependant, même si cette forme du discours scientifique est dominante, il est intéressant de s'interroger sur les autres formes de discours, celles qui se situent aux marges de la communication scientifique écrite, et en particulier celles qui peuvent ouvrir la voie de la mise en cause ou du moins de l'affinement ou de l'évolution des paradigmes. Parmi celles- ci, et malgré le développement d'Internet et le recours à l'échange de courriers électroniques entre chercheurs, les Letters to the Editor représentent un mode d'expression bien vivant dans les revues scientifiques et médicales. L'absence de validation des échanges électroniques par la communauté scientifique relègue cette forme de communication au statut de simple échange d'informations entre deux parties. Ces échanges électroniques ne peuvent donc être actuellement envisagés comme solution alternative aux Letters to the Editor qui, elles, 
ne sont publiées qu'après accord de la communauté par le processus normal de soumission aux reviewers de la revue visée.

3 L'idée de cette étude nous a été suggérée par un collègue scientifique qui nous a rapporté avoir écrit une Letter to the Editor quand il était encore jeune chercheur pour apporter la controverse à un article publié dans une revue d'écologie. Il se trouve que cette intervention au sein de sa communauté de spécialistes est désormais la référence la plus citée de cet auteur dans les revues de sa spécialité. Cet exemple conduit donc à s'interroger sur la stratégie de positionnement et de reconnaissance des auteurs de Letters to the Editor au sein des communautés de spécialistes, et soulève la problématique suivante : cette correspondance peut-elle constituer à elle seule une étape de validation de la recherche? Pour ce faire nous avons tout d'abord analysé la représentation de ce mode d'expression scientifique dans une communauté de chercheurs francophones à l'aide d'une enquête réalisée sous forme de questionnaire. Les résultats de ce questionnaire permettent de dégager les stratégies d'utilisation du genre et conduisent à proposer quelques pistes pouvant aboutir à la caractérisation du genre.

\section{Enquête sur les pratiques d'une communauté de chercheurs face à l'utilisation des Letters to the Editor}

\subsection{Pourquoi?}

4 Le corpus sur lequel nous avons travaillé est composé des Letters to the Editor publiées dans deux revues scientifique et médicale: The American Journal of Clinical Nutrition (AJCN) et The Lancet, sur une période de trois ans (1999-2001). Bien que ces revues comportent, comme toutes les revues scientifiques, une proportion importante d'articles de recherche rédigés par des chercheurs non anglophones, notre corpus est essentiellement constitué de Letters to the Editor rédigées par des chercheurs anglophones ou tout au moins travaillant dans des laboratoires de pays anglophones. Devant cette constatation, l'élargissement du corpus est apparu nécessaire. Malgré la recension d'un certain nombre de lettres rédigées par des chercheurs originaires de pays non anglophones (Italie, Amérique du Sud, Allemagne), nous n'avons relevé aucune lettre signée par une équipe française. Cette constatation ne vaut que pour les deux revues de notre corpus sur les trois années choisies, mais conduit cependant à s'interroger: les chercheurs francophones ne rédigent-ils quasiment jamais de Letters to the Editor? Ou bien leurs lettres sont-elles systématiquement refusées? Quelles sont leurs motivations quand ils décident toutefois d'écrire au rédacteur en chef d'une revue? Enfin, en termes de compétence linguistique, quel investissement cela requiert-il par rapport à l'article de recherche? Il nous a donc paru indispensable de vérifier l'utilisation de ce genre, auprès des communautés avec lesquelles nous travaillons, au moyen d'un questionnaire.

\subsection{Comment?}

5 Le questionnaire a été construit selon trois objectifs principaux autour de 24 items.

6 Tout d'abord, les quatre premières questions visent à appréhender l'utilisation passive des Letters to the Editor, c'est-à-dire leur lecture. Il s'agit de mesurer l'importance accordée à ces lettres par le temps qu'y consacrent les chercheurs ainsi que par les stratégies qu'ils développent dans le choix des revues et la fréquence de leur lecture. 
7 Ensuite les douze suivantes tentent de cerner l'utilisation active, à savoir la rédaction de courrier aux revues : les raisons motivant cette initiative, puis l'acceptation ou le refus du comité de lecture de la revue, les pratiques de rédaction en anglais et l'attente nourrie par rapport à ces courriers.

8 Enfin, les huit derniers items du questionnaire ont pour but de mesurer l'impact de cette correspondance sur le fonctionnement interne des communautés de spécialistes et sur la carrière des chercheurs à travers l'expérience des scientifiques interrogés.

\subsection{Quelles communautés ?}

Ce questionnaire a été envoyé à la communauté médicale (Professeurs des UniversitésPraticiens Hospitaliers et Assistants-Chefs de Clinique) ainsi qu'à la communauté des biologistes de l'UFR des Sciences de la Vie, du CNRS, de l'INRA et de l'ENSBANA appartenant ou étant rattachés à l'Université de Bourgogne. Le questionnaire a été envoyé à 220 chercheurs en un seul envoi le 15 décembre 2001. À la date du 31 janvier 2002, nous avions recueilli 101 réponses, ce qui a été jugé satisfaisant et suffisamment représentatif, puisque cela représente $45 \%$ des personnes interrogées. Nous avons alors commencé notre analyse.

\subsection{Résultats et analyse}

\subsubsection{Lecture}

10 Nous avons tout d'abord cherché à savoir si la lecture des Letters to the Editor représente une pratique courante dans l'activité des chercheurs. Une grande majorité ( $84 \%)$ déclare en lire non pas systématiquement mais régulièrement, et ceci, dans une ou plusieurs revues. Il s'agit pour les scientifiques de se tenir au courant de ce qui fait débat dans la spécialité, de prendre connaissance des polémiques du moment de manière rapide et efficace. Les chercheurs précisent qu'ils ne lisent pas systématiquement le courrier de la revue dans laquelle l'un de leurs articles a été publié pour en mesurer l'impact ou les réactions polémiques que celui-ci pourrait susciter. Lorsqu'ils lisent des Letters to the Editor, ils se livrent plutôt à un balayage rapide des nouveaux courants de pensée dans le seul but de se tenir informés.

\subsection{2. Écriture et publication}

11 On observe que $56 \%$ des chercheurs interrogés ont déjà eu recours à ce type de publication et ont envoyé une (20\%), deux (14\%) ou plusieurs lettres (22\%). Il est à noter que $43 \%$ des lettres envoyées sont publiées, ce qui démarque la publication d'une Letter to the Editor de celle de l'article de recherche (5\% à $10 \%$ d'acceptation dans The Lancet $\left.{ }^{1}\right)$. En revanche, le délai de publication est beaucoup plus court (un à deux mois dans un cas sur deux, et trois mois ou plus dans l'autre cas). Lorsque la lettre est refusée (tableau 1), la raison invoquée le plus souvent est le manque d'intérêt pour la revue, ce qui peut cacher bien d'autres motifs moins politiquement ou scientifiquement corrects. 
Tableau 1 : Motif de refus de la publication des Letters to the Editor

\begin{tabular}{|l|l|l|l|}
\hline $\begin{array}{l}\text { Pas } \\
\text { justification }\end{array}$ & $\begin{array}{l}\text { Manque d'intérêt pour la } \\
\text { revue }\end{array}$ & $\begin{array}{l}\text { Mauvais } \\
\text { anglais }\end{array}$ & $\begin{array}{l}\text { Pas assez critique vis-à-vis de } \\
\text { l'article }\end{array}$ \\
\hline $10,4 \%$ & $59,6 \%$ & $21 \%$ & $9 \%$ \\
\hline
\end{tabular}

12 L'autre reproche majeur porte sur la mauvaise qualité de la langue $(21 \%)$, ce qui interpelle le formateur en langue de spécialité et conduit à s'interroger sur la nécessité d'une formation linguistique adaptée à ce type d'écrit. Il faut prendre en compte, aussi bien la compétence linguistique propre (qualité de l'anglais), que la maîtrise des caractéristiques discursives et stylistiques spécifiques au genre. Enfin certains reviewers refusent de publier les lettres dans $9 \%$ des cas lorsque la remise en cause de l'article ne leur parait pas suffisamment argumentée.

13 Il est intéressant de noter que $47 \%$ des auteurs francophones interrogés font état de leur difficulté et de leur réticence à rédiger ce type d'écrit scientifique en anglais et $48 \%$ parmi les auteurs de lettres avouent s'être inspirés du style du courrier déjà publié dans la revue visée, l'utilisant comme une sorte de modèle. Cependant, la rédaction directe en anglais est largement majoritaire et seuls $6 \%$ d'irréductibles ont recours à la traduction. Ce chiffre semble conforme à ce que nous observons dans notre pratique de correcteurs/ traducteurs d'articles de recherche. Le fait d'avoir à rédiger la lettre en anglais n'est pas considéré comme un obstacle insurmontable. Les scientifiques interrogés jugent ce type d'écrit aussi difficile à rédiger qu'un article de recherche (40\%), voire moins difficile (50 \%). Cette dernière estimation est peut-être à interpréter comme un investissement en temps moins important. La rédaction d'une lettre d'une page est moins contraignante que celle d'un article d'une dizaine de pages. Si ce type d'écrit ne pose pas de problème majeur aux scientifiques, il reste à comprendre pourquoi la moyenne des chercheurs francophones a moins recours à ce type d'écrit que la communauté scientifique internationale.

\subsubsection{Interprétation des résultats}

14 Les objectifs des auteurs sont divers, mais il s'agit en priorité, dans $65 \%$ des cas (tableau 2) d'apporter un complément d'information ou une mise au point. La contradiction, la réfutation et la mise en cause de protocoles représentent environ le quart de ces interventions. En effet, le rédacteur en chef et le comité de lecture de la revue sont probablement plus réticents à publier une lettre qui vient contredire des articles publiés, et ceci en particulier lorsque la contestation est exprimée par de jeunes chercheurs. La réfutation d'un paradigme doit s'appuyer sur le fait scientifique construit, et non seulement sur une opinion formulée lors d'un courrier. Cependant, ceci ne signifie pas qu'il est impossible pour un jeune chercheur d'utiliser ce mode de communication. Lorsque cette stratégie aboutit, comme dans le cas de l'exemple cité en introduction, elle permet au chercheur de se faire une niche au sein de la communauté. Enfin, certaines lettres font figure de mini-articles de recherche en rapportant des faits nouveaux étayés par des expériences. 
Tableau 2. But de la rédaction d'une Letter to the Editor par les chercheurs francophones

\begin{tabular}{|l|l|l|l|l|l|}
\hline $\begin{array}{l}\text { Complément } \\
\text { d'information } \\
\text { point }\end{array}$ & $\begin{array}{l}\text { Mise au } \\
\text { Contradiction }\end{array}$ & Réfutation & $\begin{array}{l}\text { Mise en cause des } \\
\text { protocoles } \\
\text { expérimentaux, de la } \\
\text { méthodologie, et de la } \\
\text { formulation des } \\
\text { concepts }\end{array}$ \\
\hline $\begin{array}{l}\text { Demande } \\
\text { d'information } \\
\text { supplémentaire }\end{array}$ & $\begin{array}{l}\text { Rapport de cas } \\
\text { exemplaire clinique }\end{array}$ & Rapport préliminaire \\
\hline $1,5 \%$ & $5,5 \%$ & $7 \%$ \\
\hline
\end{tabular}

Parmi les chercheurs qui ont obtenu la publication d'une ou plusieurs lettres, la moitié environ n'a pu mesurer de manière précise une forme quelconque de retombée (tableau 3). L'autre moitié estime que leurs lettres ont suscité un intérêt variable allant d'un simple échange de lettres ou e-mails (13\%) à l'accroissement de leur notoriété au sein de leur communauté et à l'intégration de cette lettre dans leur liste de publications $(8 \%)$. Pour $7 \%$ de chercheurs, publier une lettre est un moyen efficace d'affiner la recherche, en particulier la méthodologie.

Tableau 3. Estimation des retombées de la publication d'une Letter to the Editor par les chercheurs

\begin{tabular}{|l|l|l|l|l|l|}
\hline $\begin{array}{l}\text { Aucune } \\
\text { pas de } \\
\text { retour }\end{array}$ & $\begin{array}{l}\text { Faible } \\
\text { très } \\
\text { modeste }\end{array}$ & $\begin{array}{l}\text { Échange de } \\
\text { lettres, d'e- } \\
\text { mails }\end{array}$ & $\begin{array}{l}\text { Notoriété dans la } \\
\text { communauté }\end{array}$ & $\begin{array}{l}\text { Lettre } \\
\text { régulièrement } \\
\text { citée }\end{array}$ & $\begin{array}{l}\text { Affinement de } \\
\text { la recherche }\end{array}$ \\
\hline $56 \%$ & $16 \%$ & $13 \%$ & $4 \%$ & $4 \%$ & $7 \%$ \\
\hline
\end{tabular}

Si une majorité de chercheurs (64\%) estime que la publication d'une ou de plusieurs Letters to the Editor n'a pas entraîné de retombées significatives sur leur propre carrière (tableau 4), en revanche, la moitié des scientifiques interrogés pense que cet échange de correspondance, validé par les comités de lecture des revues, joue un rôle non négligeable au sein de leur communauté.

Tableau 4 : Estimation de l'impact de la publication d'une Letter to the Editor sur la communauté de spécialistes et la carrière des chercheurs

\begin{tabular}{|l|l|l|l|l|l|l|l|}
\hline \multicolumn{3}{|l|}{ Impact sur la carrière } & \multicolumn{3}{|c|}{ Impact sur la communauté de chercheurs } \\
\hline Oui & Non & Ne sait pas & Pas de réponse & Oui & Non & Ne sait pas & Pas de réponse \\
\hline $10 \%$ & $64 \%$ & $12 \%$ & $14 \%$ & $50 \%$ & $22 \%$ & $10 \%$ & $18 \%$ \\
\hline
\end{tabular}



scientifiques mis en place par des instances telles que le CNU ou le CNRS qui prennent essentiellement en compte les articles canoniques, publiés dans les revues classées par le SCI (Science Citation Index) et dans lesquelles les Letters to the Editor ne sont pas identifiées comme publications à part entière. C'est entre autres à ce niveau que la discrimination entre jeunes chercheurs et chercheurs confirmés est la plus perceptible. L'engagement personnel du chercheur, marqué par la forte prise en charge de l'énoncé, au sens linguistique du terme, et la signature personnelle, conduit les comités de lecture à favoriser la publication de lettres de chercheurs déjà réputés dans la communauté. Pour un jeune chercheur, la publication d'une lettre doit s'appuyer sur un élément majeur permettant la remise en cause d'articles validés par la communauté.

Un fait certain aux yeux de la communauté scientifique est qu'une simple lettre publiée dans une revue possédant un impact important peut être l'une des premières étapes de la remise en cause d'un paradigme dominant. En effet, pour $64 \%$ des chercheurs, c'est le moyen d'engager le dialogue avec une équipe concurrente, et selon $50 \%$ des chercheurs sondés, ce genre d'intervention peut influencer le positionnement de certains chercheurs ou de la communauté par rapport à un paradigme. auteurs des lettres ont déjà acquis une solide réputation parmi les spécialistes. Ce mode relationnel est jugé satisfaisant et efficace, car il permet d'informer rapidement et de manière ciblée plus de monde qu'une simple communication en congrès. Il peut aboutir au (re)positionnement d'une équipe, il permet également de prendre date avant une publication plus conséquente. Il permet enfin à certains chercheurs de justifier a posteriori un protocole expérimental critiqué par leurs pairs.

En revanche, un petit nombre déplore le caractère partiel et partial de ce type de communication, dénonce son côté polémique et stérile, lui préférant l'aspect direct et plus convivial des échanges électroniques et des séances de questions-réponses lors des congrès.

21 En conclusion, $66 \%$ des auteurs de lettres déclarent inclure les lettres publiées dans leur liste de titres et travaux, mais celles-ci ne sont pas intégrées parmi les articles de recherche et ne figurent pas parmi les publications majeures de leur CV. Il ne faut pas oublier que $44 \%$ du panel de chercheurs francophones interrogés n'ont jamais soumis de Letter to the Editor. Ceci peut être interprété, soit comme une absence d'intérêt pour ce genre de communication, soit comme une méconnaissance et donc un manque de maîtrise du genre. Il faut se rappeler le temps qui a été nécessaire à la communauté scientifique francophone pour se familiariser avec les canons de l'article de recherche en anglais et se les attribuer. Il semblerait que la même démarche soit nécessaire pour les autres formes de communication scientifique, dont les Letters to the Editor. Ceci conduit donc à s'interroger sur les caractéristiques formelles et culturelles de ces lettres afin d'en dégager un éventuel modèle, ce qui permettrait de conclure qu'il s'agit bien d'un genre identifiable et reproductible. 


\section{Les Letters to the Editor peuvent-elles être considérées comme un genre modélisable?}

22 Notre enquête a permis de mieux cerner le rôle de ce genre de discours au sein de la communauté scientifique, de montrer que les chercheurs francophones (médecins ou biologistes) lisent régulièrement cette correspondance, mais n'ont pas toujours recours à ce genre eux-mêmes. Notre rôle est de chercher à savoir pourquoi. Nous avons évoqué le manque de reconnaissance au niveau du CNU ou du CNRS. Vient ensuite la difficulté de rédiger en anglais un type d'écrit qui pour l'instant est moins modélisé ou modélisable.

\subsection{Caractéristiques communes avec l'article de recherche}

23 Étant donné que l'article de recherche est la forme la mieux maitrisée par les scientifiques et la forme la plus étudiée par les linguistes, il semble opportun de comparer ces deux modes d'expression pour en déduire les caractéristiques propres du genre et permettre ainsi son analyse.

\subsubsection{Existence de Guidelines}

Au même titre que l'article de recherche, certaines revues ont progressivement élaboré des guidelines. Elles fixent la longueur maximale, le délai de réaction par rapport à l'article concerné, et la nature des thèmes qui peuvent être abordés. Les lettres peuvent être modifiées sans accord préalable de l'auteur afin d'apporter plus de lisibilité et de concision. Elles peuvent apparaître dans la revue papier et/ou en ligne.

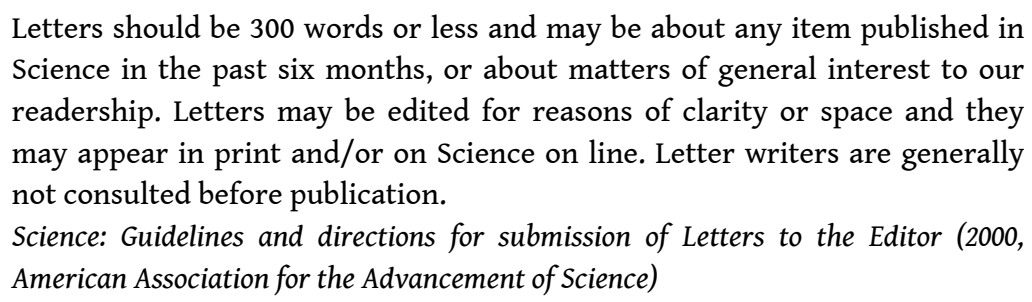
Science in the past six months, or about matters of general interest to our readership. Letters may be edited for reasons of clarity or space and they may appear in print and/or on Science on line. Letter writers are generally not consulted before publication.

Science: Guidelines and directions for submission of Letters to the Editor (2000, American Association for the Advancement of Science)

Il faut noter cependant qu'aucune consigne n'est donnée concernant l'organisation argumentative, le style, la formulation des critiques. D'autre part, les critères retenus par la revue pour accepter ou refuser la publication d'une lettre sont peu explicités.

\subsubsection{Un genre identifiable, évolutif avec macrostructure sous-jacente}

Les Letters to the Editor sont apparues dans les années 1920 dans les revues scientifiques sélectionnées pour notre corpus, et cette pratique s'est accrue avec le temps. Il semble que l'objectif des auteurs se soit progressivement inscrit dans la stratégie générale de publication, du moins pour les chercheurs anglophones confirmés.

27 En effet, pour ceux-ci, la rédaction d'une lettre peut apparaître comme un exercice aisé, même lorsque celle-ci doit tenir compte du style propre à chaque revue. En revanche, pour les chercheurs francophones, cette pratique s'apparente à un exercice de style avec lequel ils sont peu familiarisés. Notre but est donc de disséquer ce mode d'expression, de 
cerner les différents genres auxquels ce type d'écrit peut s'apparenter pour le rendre plus abordable à nos communautés de chercheurs.

Après avoir déjà décrit quelques caractéristiques linguistiques (Magnet \& Carnet, 2001 : 51-62), nous nous proposons d'aborder les principaux traits discursifs inhérents à la macrostructure. Le schéma argumentatif le plus récurrent peut être décrit selon quatre mouvements en empruntant à J. Swales (1981) son approche descriptive (tableau 5).

Tableau 5. Proposition de macrostructure des Letters to the Editor

\begin{tabular}{|l|l|l|}
\hline & Stratégie & Exemples de formulation \\
\hline $\begin{array}{l}\text { Move } \\
1\end{array}$ & $\begin{array}{l}\text { Rappel des résultats publiés } \\
\text { contestés }\end{array}$ & $\begin{array}{l}\text { We read with interest the work by... } \\
\text { The recent study of [...] examined the association } \\
\text { between... }\end{array}$ \\
\hline $\begin{array}{l}\text { Move } \\
2\end{array}$ & $\begin{array}{l}\text { Formulation du problème qui } \\
\text { suscite la mise en cause }\end{array}$ & $\begin{array}{l}\text { The report by Kraaijenhagen and colleagues adds } \\
\text { nothing to the debate... } \\
\text { I believe that their study was underpowered to } \\
\text { reach this conclusion. }\end{array}$ \\
\hline 3 & $\begin{array}{l}\text { Argumentation appuyée sur les } \\
\text { recherches de l'auteur de la lettre }\end{array}$ & $\begin{array}{l}\text { Yet, we showed no significant decrease... } \\
\text { First and foremost, we feel that an important } \\
\text { aspect of our studies [...] was missed... }\end{array}$ \\
\hline $\begin{array}{l}\text { Move } \\
4\end{array}$ & $\begin{array}{l}\text { Conclusion et forte incitation à } \\
\text { revoir les résultats initiaux }\end{array}$ & $\begin{array}{l}\text { Given the distinguished provenance of this wisdom, } \\
\text { issue now. } \\
\text { It is time for some UK doctors to take their heads } \\
\text { out of the sand ... }\end{array}$ \\
\hline
\end{tabular}

La première étape consiste à rappeler les résultats publiés incriminés. Ce rappel se fait par des formulations telles que :

The recent article in the Journal by Fernandez et al pointing out that [...] is an important work that opens the door to increased prevention of such cancers. (AJCN, 71, 2000:599)

We read with interest the work by... (AJCN, 72, $2000: 199$ )

The recent study of [...] examined the association between... (AJCN, 72, 2000 : 1059)

La seconde étape s'attache à formuler le problème qui suscite la mise en cause. Cette formulation apparait dans des tournures comme :

The report by Kraaijenhagen and colleagues adds nothing to the debate on travel risk and DVT. The study was poorly designed, retrospective and biased. (The Lancet, 357, 2001 : 553)

The method they use does not seem to be suited to the end-result. [...] I believe that their study was underpowered to reach this conclusion. (The Lancet, 357, $2001: 553$ ) 
This finding is in contrast with the recent heart outcomes prevention evaluation study in which vitamin E seemed to have no significant effect in patients at high risk of cardiovascular disease. (The Lancet, 357, $2001: 631$ ) recherches de l'auteur de la lettre :

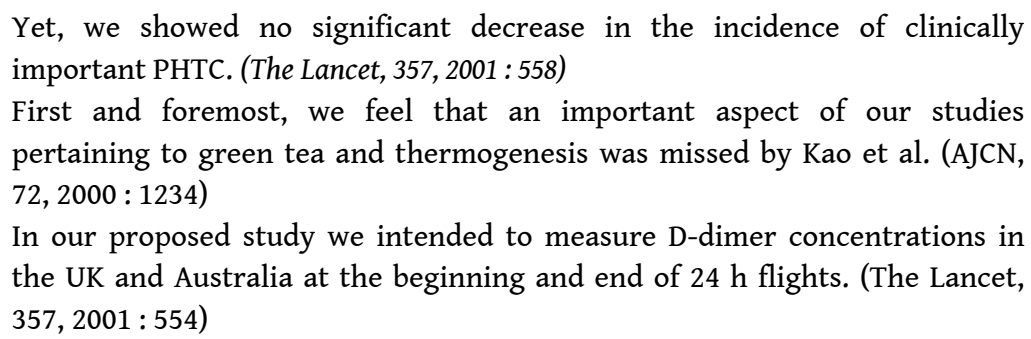

Enfin, la dernière étape incite fortement, lors de la conclusion, à revoir les résultats initiaux de l'étude contestée :

Given the distinguished provenance of this wisdom, it is truly urgent that nutritionists resolve this issue now. (AJCN, 1999 : 70)

We invite Lowe to join us in making this a reality for people living in poorer countries. (The Lancet, 357, $2001: 724$ )

Clarification of the above statement may be necessary to prevent confusion and to avoid detracting interest from a potentially important risk factor for sarcopenia. (AJCN, 71, 2000 : 1209)

It is time for some UK doctors to take their heads out of the sand. (The Lancet, 357, $2001: 555$ )

Bien que ce schéma ne soit pas systématique, il est donc possible de conclure à une forme semimodélisée de la construction argumentative des Letters to the Editor.

\section{Caractéristiques propres}

Contrairement aux apparences, les Letters to the Editor n'appartiennent pas au genre épistolaire. Comme dans toute publication scientifique, la communication s'instaure entre un scientifique et sa communauté et non entre deux individus. Ce ne sont donc pas de véritables lettres mais le moyen d'exprimer des points de vue, des prises de position.

Il faut donc les rattacher aux «lettres ouvertes» qui appartiennent au style journalistique et non à l'écriture intime, caractéristique du genre épistolaire.

\subsection{Un genre centré sur le chercheur (researcher centered)}

Par rapport à l'article de recherche dont la raison d'être est le fait scientifique, dans les Letters to the Editor, la focalisation porte davantage sur les chercheurs, leur position dans la communauté, et le désaccord sur un argument scientifique peut parfois tourner au conflit d'intérêts. Par le biais de l'article de recherche, il pourrait sembler que le seul intérêt des chercheurs est de faire avancer la science, alors que par celui des Letters to the Editor, les enjeux et les conflits sous-jacents deviennent explicites. L'article de recherche est centré sur la recherche (research centered) alors que les Letters to the Editor sont centrées sur les chercheurs (researcher centered). 


\subsection{Spécificités culturelles}

\subsubsection{Recours au « politiquement correct » dans les revues américaines}

Les deux revues composant notre corpus se distinguent nettement par le ton adopté et notamment par le recours au " politiquement correct » dans la revue américaine. Dans la communauté scientifique américaine, la notion du "politiquement correct " consiste à critiquer essentiellement l'objet (l'expérience, la méthodologie sélectionnée) et non le sujet (l'équipe de chercheurs). De plus, cette attitude conduit les auteurs de lettres à pratiquer l'euphémisme et à utiliser fortement la technique du hedging, c'est-à-dire à recourir à des formes modalisées :

Finally Bray and Popkin's conclusion about energy density must be regarded as an interesting but unproven hypothesis... I suspect the hypothesis may prove to be overly simplistic. (AJCN, 70, $1999: 573$ )

Perhaps a more pertinent study would be one that investigates why editors feel compelled to challenge not only the management of obesity but the very existence of the disease itself. (AJCN, 71, $2000: 845$ )

En revanche, The Lancet, revue britannique, abonde en formes dont l'aspect polémique apparaît de manière évidente, allant jusqu'à l'expression de l'agressivité, voire du sexisme :

\footnotetext{
I think that Sandra Simkin's report is inappropriate, inaccurate, and emotive. (The Lancet, 357, $2001: 641$ )

The study was poorly designed, retrospective, and biased. (The Lancet, 357, $2001: 553)$
}

La critique ouverte porte directement sur la personne et illustre à la fois le caractère " researcher centered » que l'on vient de mentionner et l'absence de retenue policée chez les Britanniques.

\subsubsection{Références culturelles}

Certaines lettres portent un titre qui ne pourrait en aucun cas se trouver dans l'article de recherche. Ces titres font appel à un fond culturel réputé commun à l'ensemble des chercheurs, en réalité celui-ci est essentiellement anglo-saxon. La référence peut être culturelle, humoristique ou autre. Voici quelques exemples relevés dans notre corpus.

\subsubsection{Citations bibliques}

Dans certains cas, les auteurs anglophones déguisent leurs critiques acerbes sous des emprunts culturels en détournant par exemple une citation biblique. Même si la Bible appartient au fond culturel universel, il est bien connu que celle-ci imprègne davantage le monde anglo-saxon. Il est donc bien difficile pour un scientifique non anglophone de comprendre l'allusion et encore plus difficile pour celui-ci de détourner le culturel pour exprimer une critique sous une telle forme.

Seek and thou shalt find

Patino et al doubt the presence of endotoxin in their study cohort, although we know it to be present in the general population. 
Higher endotoxin levels in patients with diabetes mellitus and arterial disease may be expected and should be sought (AJCN, 86, $2000: 1054)$

\title{
Ask and it shall be given you; seek and ye shall find ", Matthieu, VII, 7, Luc, XI, 9], se révèle
} particulièrement critique à l'égard de la méthodologie développée par l'équipe en cause.

\subsubsection{Néologismes}

recours à des néologismes facétieux pour montrer l'inadéquation d'une terminologie courante dans la communauté scientifique peut prêter à sourire. Par exemple, l'utilisation du terme vegetarian considéré comme trop vague et imprécis est critiquée. Il pousse les auteurs à s'amuser à faire des variations loufoques sur le thème.

\begin{abstract}
In an attempt to add more specificity in the scientific literature, various qualifying terms for vegetarian have been used, such as pescovegetarian and lactoovovegetarian. For one person I know who considers himself a vegetarian, an appropriate label might be lactoovopescopoulosteak-onlywhen-I-eat-out vegetarian. (AJCN, 71, 2000 : 1211)
\end{abstract}

Il s'agit en réalité d'une critique humoristique de l'usage d'une terminologie de vulgarisation par une communauté qui d'ordinaire revendique un lexique spécialisé.

\subsubsection{Citations populaires}

Certaines citations apparaissent sous la forme d'aphorismes :

« Small is beautiful »: $\alpha$-linolenic acid and eicosapentaenoic acid in man

L'explicitation est fournie dans la conclusion de la lettre :

Minor changes in dietary lipids may be most significant in thrombosis prevention. (The Lancet, 1999 : 1169)

Là encore, l'auteur de la lettre critique le manque de perspicacité de ses collègues auteurs de l'article qui n'ont pas su tirer les conséquences d'un changement en apparence mineur.

D'autres empruntent au registre de la chanson:

Mechanical circulatory support - a long and winding road (AJCN, 71, 2000 : 1222)

Toutes ces techniques faisant appel au registre de l'humour et de l'ironie, par le biais du détournement de formules empruntées à d'autres genres, s'avèrent impraticables par des chercheurs francophones. Le recours à un fond culturel commun non scientifique est une des caractéristiques des Letters to the Editor, au contraire de l'article de recherche, et peut expliquer la quasi-absence de lettres rédigées par les francophones dans notre corpus.

\section{Conclusion}

50 Cette étude permet de mieux cerner le rôle de ce genre au sein de la communauté scientifique. Il faut noter que les chercheurs francophones (médecins ou biologistes) 
lisent des Letters to the Editor, mais n'ont pas toujours recours à ce genre eux-mêmes. Les références culturelles fréquentes peuvent en partie justifier leurs réticences. En l'état actuel de nos recherches, nos conclusions sont les suivantes.

51 Il n'existe pas de différence majeure dans la stratégie de lecture et de rédaction des Letters to the Editor entre biologistes et médecins francophones, même si sur le plan quantitatif, on trouve davantage de lettres publiées dans les revues de médecine que dans les revues de biologie.

52 Les différences dans l'utilisation du genre se font plutôt sentir entre jeunes chercheurs et chercheurs confirmés. Ceux-ci recherchent davantage la consolidation de leur position au sein de la communauté internationale, alors que leurs collègues plus jeunes ont le besoin impérieux de prouver, par des articles de recherche, qu'ils sont productifs.

53 Même lorsque les scientifiques ont fréquemment recours à ces lettres, elles ne suffisent pas pour se construire une réputation de chercheur de haut niveau. On peut les considérer comme utiles, voire nécessaires, mais non suffisantes. Elles reflètent les tensions au sein de la communauté. Elles font état des conflits et des désaccords.

Elles ne représentent généralement pas une forme authentique de validation par la communauté scientifique. La communauté ne s'engage pas, ne prend pas parti, et le comité de lecture se réserve le droit de publier ou non les lettres soumises, et même de les transformer.

Le rôle le plus intéressant semble être celui de « soupapes ", permettant aux chercheurs d'exprimer frustrations, oppositions, désaccord, regrets... Les Letters to the Editor paraissent jouer un rôle libérateur permettant au chercheur de se dégager de l'aspect formel et impersonnel que lui impose l'article de recherche pour adopter une prise de position plus marquée et moins nuancée.

56 Ces lettres sont moins modélisées que l'article scientifique, elles offrent donc un espace de liberté d'expression au chercheur et le moyen d'échapper à la lourdeur des publications plus canoniques et traditionnelles du discours scientifique.

57 Enfin, un rôle essentiel est celui de vivier pour de nouvelles recherches en fournissant l'inspiration, d'autres pistes à explorer, ou des perspectives de collaborations nouvelles. Il serait donc intéressant, lors d'une étude ultérieure, de comparer le poids de cet échange au sein de la communauté, par rapport à l'échange d'e-mails, de forums et de la session de questions-réponses lors des congrès scientifiques.

\section{BIBLIOGRAPHIE}

Magnet, Anne \& Didier Carnet. 2001. «Quelques aspects de la contradiction et de la remise en cause dans le genre Letters to the Editor ». ASp 31-33, 51-62.

Martin, Jacky. 1996. «Les enjeux du discours scientifique : la stratégie de véridiction ». ASp 11-14, 13-31. 
Swales, John. 1981. Aspects of Article Introductions. Birmingham : The University of Aston, Language Studies Unit.

Swales, John. 1990. Genre Analysis. Cambridge : Cambridge University Press.

\section{Bibliographie}

Behe, M. J. 2002. «Correspondence with science journals: Response to critics concerning peerreview ». In Access Research Network <http://www.arn.org/docs/behe/ $\mathrm{mb} \_$correspondencewithsciencejournals.htm>.

Berthoud, A. \& L. Mondada (dir.). 2000. Modèles du discours en confrontation. Berne, Berlin : Peter Lang.

Charolles, M., S. Fisher \& J. Jayez (dir.). 1990. Le discours, représentation et interprétations. Nancy : Presses Universitaires de Nancy.

Cherchi, L. 1983. «Sur la valeur explicative de la notion d'engagement ». Modèles linguistiques V/1, 63-80.

Crosnier, E. 1994. « Enquête sur l'évaluation de l'anglais par les Anglophones dans les publications des scientifiques français. Critères linguistiques, résultats et perspectives ». ASp 3, 41-55.

Hacking, I. 1985. «Styles of scientific reasoning ». In Rajchman J. \& C. West (dir.), Post Analytic Philosophy. New York : Columbia University Press, 145-165.

Halliday, M.A.K. \& J.R. Martin. 1993. Writing Science, Literary and Discursive Power. Pittsburg, Pennsylvania : University of Pittsburg Press.

Harnad, S. 1983. Peer Commentary on Peer Review: A Case Study in Scientific Quality Control. Ann Arbor, MI : Books on demand UMI.

Latour, B. 1993. We Have Never Been Modern. Cambridge, MA : Harvard University Press.

Lynch, M. 1993. Scientific Practice and Ordinary Action: Ethnomethodology and Social Studies of Science. Cambridge : Cambridge University Press.

Magnet, A. 1993. « La discussion de l'article scientifique : quelques aspects linguistiques et discursifs dans trente-six articles de nutrition ». Mémoire de DEA, Bordeaux.

Mel'cuk, I. 1997. Vers une linguistique sens-texte. Leçon inaugurale au Collège de France, chaire internationale. Paris : Collège de France éditeur.

Meldrum, J. M. 2002. " The curious affair of the Edinburgh international science festival invitation ». <http://www.freenetpages.co.uk/hp/a.pusztai/sci-fest.htm>.

Mémet, M. 1993. « Le premier DEA de langue anglaise des spécialités scientifiques et techniques : contraintes, libertés ». ASp 2, 230-257.

Myers, G. A. 1989. « The pragmatics of politeness in scientific articles ». Applied Linguistics 10, 1-35.

Smolin, L. 2002. « Storming the ivory tower ». New Scientist 2332, 40-43.

\section{NOTES}

1. Le pourcentage d'acceptation nous a été communiqué par le rédacteur en chef de la revue dans un courrier du 29 janvier 1998. 


\section{RÉSUMÉS}

Cet article se fixe comme objectif d'analyser la fonction et l'utilisation du genre Letters to the Editor au sein d'une communauté de chercheurs francophones. L'impact et la représentation de ce mode d'expression scientifique ont été mesurés à l'aide d'une enquête sous forme de questionnaire dont les résultats sont rapportés et analysés. Cette étude permet de dégager les stratégies d'utilisation du genre et conduit à proposer quelques pistes pouvant aboutir à la caractérisation du genre. Ce travail s'appuie également sur l'analyse comparée d'un corpus (1999-2001) composé à partir d'une revue de biologie et d'une revue médicale.

The aim of this paper is to analyse the goal, role and use of the genre Letters to the Editor within a community of French researchers. The impact and representation of this mode of scientific expression were measured through a survey based on a questionnaire whose results are reported and analysed. This study brings to the fore the strategies underlying the genre and suggests certain avenues to explore which may lead to the characterization of the genre. This work is also based on the compared analysis of a corpus (1999-2001) selected in the fields of biology and medicine.

\section{INDEX}

Mots-clés : analyse du discours, biologie, correspondance avec les journaux scientifiques, médecine, questionnaire, stratégie d'utilisation

Keywords : biology, discourse analysis, letter to the editor, medicine, questionnaire, strategy (underlying)

\section{AUTEURS}

\section{DIDIER CARNET}

Didier Carnet, agrégé d'anglais, est maître de conférences à l'Université de Bourgogne, Faculté de Médecine de Dijon. Sa recherche porte sur la linguistique et la didactique en anglais médical (enseignement initial et formation continue). Il est le coordinateur de plusieurs ouvrages : L'anglais médical à la Faculté (Ellipses 1994), Words, Sciences de la santé (Ellipses, 2001), L'anglais pour la santé : Exercices d'appropriation de la langue à visée professionnelle (Ellipses 2001) et La communication orale scientifique en anglais (Ellipses 2002). Il est également le coordinateur du CD Rom Dictionnaire de la santé (Grand bilingue, Mysoft, 2002) et il termine la préparation d'un CD Rom sur la communication orale scientifique. d.carnet@u-bourgogne.fr

\section{ANNE MAGNET}

Anne Magnet est maître de conférences à l'Université de Bourgogne, U.F.R. des Sciences de la Vie. Son domaine de recherche est l'analyse du discours scientifique et la linguistique de corpus appliquée aux langues dans le domaine de la biologie. Sa recherche récente s'est intéressée à 
l'analyse sémiologique des éléments non textuels de l'article de recherche. Ses dernières communications ont porté sur l'activité du chercheur en tant que médiateur du réel et sur les modes mineurs de la publication scientifique tels que les Letters to the Editor. anne.magnet@ubourgogne.fr 